\title{
Correlates of monoicy and dioicy in hornworts, the apparent sister group to vascular plants
}

\author{
Juan Carlos Villarreal ${ }^{*}$ and Susanne S Renner
}

\begin{abstract}
Background: Whether male and female gametes are produced by single or separate individuals shapes plant mating and hence patterns of genetic diversity among and within populations. Haploid-dominant plants ("bryophytes": liverworts, mosses and hornworts) can have unisexual (dioicous) or bisexual (monoicous) gametophytes, and today, $68 \%$ of liverwort species, $57 \%$ of moss species, and $40 \%$ of hornwort species are dioicous. The transitions between the two sexual systems and possible correlations with other traits have been studied in liverworts and mosses, but not hornworts. Here we use a phylogeny for 98 of the 200 species of hornworts, the sister group to vascular plants, representing roughly equal proportions of all monoicous and all dioicous species, to test whether transitions in sexual systems are predominantly from monoicy to dioicy as might be expected based on studies of mosses. We further investigate possible correlations between sexual system and spore size, antheridium number, ploidy level, and diversification rate, with character selection partly based on findings in mosses and liverworts.

Results: Hornworts underwent numerous transitions between monoicy and dioicy. The transition rate from dioicy to monoicy was $2 x$ higher than in the opposite direction, but monoicous groups have higher extinction rates; diversification rates do not correlate with sexual system. A correlation important in mosses, that between monoicy and polyploidy, apparently plays a small role: of 20 species with chromosome counts, only one is polyploid, the monoicous Anthoceros punctatus. A contingency test revealed that transitions to dioicy were more likely in species with small spores, supporting the hypothesis that small but numerous spores may be advantageous for dioicous species that depend on dense carpets of gametophytes for reproductive assurance. However, we found no evidence for increased antheridium-per-chamber numbers in dioicous species.
\end{abstract}

Conclusions: Sexual systems in hornworts are labile, and the higher number of extant monoicous species (60\%) may be largely due to frequent transitions to monoicy.

Keywords: Chromosome counts, Sexual systems, Spore size, Trait correlation

\section{Background}

Understanding the evolution of plant sexual systems requires a phylogenetic background and a basic knowledge of plant life cycles. All embryophytes cycle between a haploid and a diploid life stage, and in the course of evolution, the diploid stage became the dominant phase in lycophytes, ferns and seed plants, while in liverworts, mosses, and hornworts the haploid stage is the dominant phase. This cycling has numerous implications, including for the evolution of plant sexual systems. There are four basic kinds of sexual systems, namely systems in which (i) the haploid stage produces archegonia and

\footnotetext{
* Correspondence: jcarlos.villarreal@gmail.com

Systematic Botany and Mycology, Department of Biology, University of Munich (LMU), Munich, Germany
}

antheridia on each gametophyte (called monoicy); (ii) archegonia and antheridia are produced on separate gametophytes (dioicy); (iii) archegonia/embryo sacs and antheridia/microsporangia are produced on each sporophyte (monoecy); and (iv) archegonia/embryo sacs and antheridia/microsporangia are produced on separate sporophytes (dioecy; [1,2]; our Figure 1). Of these four systems, the two found in diploid-dominant plants, namely monoecy and dioecy, are not directly equivalent to the two found in haploid-dominant plants, namely monoicy and dioicy, because in haploid-dominants, the sporophyte is always monoicous, produces male and female spores (future gametophytes) in a 50:50 ratio, and is heterozygous at the sex locus, while in diploiddominants, the sporophyte can become sexually 


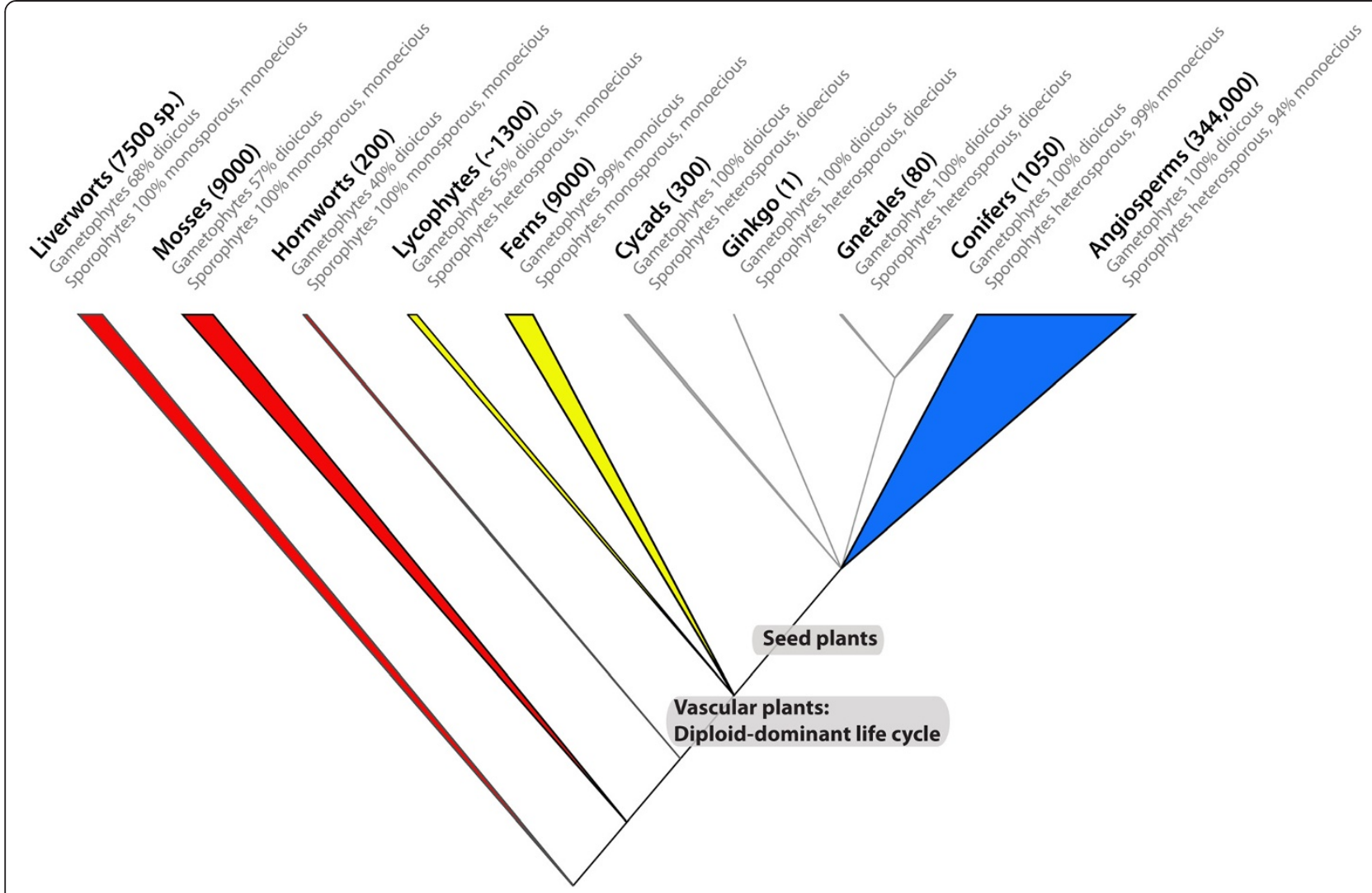

Figure 1 Distribution of the four basic sexual systems of embryophytes. The basic sexual system in embryophytes are illustrated namely, monoicy and dioicy in gametophytes (haploid organisms), and monoecy and dioecy in sporophytes (diploid organisms). Triangle width is proportional to species number and absolute species numbers are shown at the tips. The haploid-dominant lineages (liverworts, mosses, and hornworts) are shown in red triangles, the diploid-dominant lineages in yellow (lycophytes, ferns), gray (gymnosperms) and blue (angiosperms) triangles. Sources: Liverworts and mosses [2-5]; hornworts (this study); lycophytes and ferns [3,6]; http://www.rz.uni-karlsruhe.de/ db111/flora/ ferns/index.php; seed plants ([7] and this study).

specialized, then producing just one kind of spore (gametophyte), and be homozygous at the sex locus. This has evolved in cycads, Ginkgo, a few conifers (all Gnetales) and a few angiosperms (Figure 1). The frequencies of the four sexual systems in the major lineages of embryophytes vary dramatically.

Among the striking evolutionary changes along the phylogeny of embryophytes (Figure 1) is how the distribution of sexual systems changes between hornworts and vascular plants, their apparent sister group [8], partly because of the repeated evolution of heterospory, which entails sexually specialized gametophytes. Hornworts have $60 \%$ monoicous species and $40 \%$ dioicous ones, frequencies resembling those in liverworts and mosses, but the situation is completely different in ferns and fern-like lineages. In spite of their pivotal phylogenetic position, the evolution of monoicy and dioicy in hornworts has never been analyzed, probably because of the lack of a phylogenetic framework and outdated information on the presence or absence of monoicy and dioicy in the various genera. The directionality of change between monoicy and dioicy is therefore unknown. Here we use a modern hornwort phylogeny, based on plastid and mitochondrial loci and comprising 98 of the 200 species, and original data on the occurrence of monoicy and dioicy in the different species, to infer the minimal number of switches between monoicy and dioicy in this clade. We also test whether correlations between these two sexual systems and certain traits that have been detected in studies of liverworts and mosses, hold true in hornworts. We explain the background for these correlations in the next sections.

Sexual reproduction in dioicous hornworts, as in liverworts and mosses, depends on the availability of water and short distances between antheridia and archegonia, which is required for the motile sperm to fertilize the egg. Hornworts with separate-sexed gametophytes may therefore have a less assured reproduction than hornworts with cosexual gametophytes. However, intragametophyte mating is equivalent to the most extreme form of selfing, resulting in 100\% homozygous progeny in a single generation. There is some evidence for selfing 
in monoicous mosses [9-11], but no data are available for hornworts. In spite of these expectations, monoicy and dioicy occur in roughly equal proportion in all three haploid-dominant embryophyte lineages (Figure 1). This raises the question of whether other traits precondition a lineage towards one of the two sexual systems. For example, in mosses, there appears to be a correlation between dioicy and small spores [12]. This became apparent in an analysis that used grafted phylogenies ([12]: Additional file 1: Table S1) to look at correlations between sexual system, spore size, seta length, and polyploidy. (Since the phylogeny was grafted, Crawford et al. [12] only analyzed correlations but did not infer the evolutionary direction of sexual system transitions). Dioicous mosses were more likely to have small spores if spore size was coded as a discrete character, but not if it was coded as a continuous character. The underlying cause of the possible correlation between spore size and monoicy was seen in the greater dispersal distance of small spores compared to large spores, which would compensate for higher local extinction rates of dioicous species (because of reproductive failure when sperm cannot reach egg cells).

In hornworts, spore sizes range from $18 \mu \mathrm{m}$ diameter in Leiosporoceros up to $>100 \mu \mathrm{m}$ in the multicellular spores of Dendroceros [13]. If small spores are cheaper to produce than large ones they can be produced in larger numbers, which might be especially advantageous in dioicous species for which a dense spore shadow and dense population of gametophytes might provide reproductive assurance (because sperm would not have to swim far). The antheridia and archegonia of hornworts are embedded inside the thallus, and antheridium number varies from one up to 80 per chamber, with fairly narrow species-specific ranges [13,14]. Excluding possible animal vectors (such as mites), the flagellate sperm cells travel only a few centimeters [14-16]. Our initial expectation therefore was that dioicous hornworts would produce not only small spores, but also a higher number of antheridia per chamber than monoicous species to be able to produce more numerous sperm, again for reproductive assurance.

Besides testing for correlations between sexual system, spore diameter, and/or antheridium number, we were interested in whether there might be a correlation between polyploidy and sexual system. Hornworts usually have a low number of chromosomes, typically $n=5(4+\mathrm{U} / \mathrm{V}$, the presumed sex chromosomes) in dioicous species and $n=5-6$ in monoicous species, with few polyploid species [17]. Polyploidy is extremely rare in liverworts [18,19], but in mosses, polyploidy is common, and polyploid species tend to be monoicous $([2,12,20]$, and references therein). This is expected because following autopolyploidy, random pairing of the sex chromosomes will lead to both dioicous and monoicous offspring (depending on chromosome segregation), while following allopolyploidy, there will be only monoicous progeny, since after homeologous pairing each spore will contain a U chromosome and a V chromosome [2,20,21].

The species included in our phylum-wide phylogeny were selected to represent monoicous and dioicous species more or less in the proportion found across all hornworts, so as best to be able to answer the following questions: (i) Is dioicy or monoicy the more likely sexual system during the early phase of hornwort evolution? (ii) To what extent are shifts in sexual system correlated with shifts in spore size, antheridium number, and/or polyploidy? And (iii) is dioicy associated with lower diversification rates?

\section{Methods}

Taxon sampling, isolation of DNA, amplification, and sequencing

We sampled 98 of the 200 species of hornworts; Additional file 1: Table S1 provides a list of the sampled species with taxonomic author names, herbarium vouchers, and GenBank accession numbers for all sequences. Determination of plant material relied mostly on comparison with type material, but five of the sampled species are not yet formally described.

DNA isolation followed standard protocols. To deduce phylogenetic relationships we used the mitochondrial nad5-exon2, excluding an intron of $~ 950$ nucleotides that is unique to Leiosporoceros, Anthoceros, Folioceros and Sphaerosporoceros [22] the plastid gene $r b c \mathrm{~L}$ and portions of the trnK intron and the matK gene contained within it (primers designed by Alan Forrest, Royal Botanical Garden Edinburgh). Total DNA from fresh, silica-dried or herbarium material was extracted with the Nucleo-Spin plant kit according to the manufacturer's protocol (Macherey-Nagel, Düren, Germany). Primers and standard PCR protocols are listed in [23] except primers newly designed for mat $^{\mathrm{a}}$. PCR products were cleaned using ExoSap-it (Affymetrix, Santa Clara, CA, USA), and sequencing reactions using Big Dye version 3.1 were run on an ABI 3130 capillary sequencer (Applied Biosystems, Perkin-Elmer, Wellesley, MA, USA), following manufacturers' protocols. Sequence editing and alignment were carried out in Sequencher 4.7 (Gene Codes, Ann Arbor, MI, USA) and Geneious v. 5.6.6.

\section{Phylogenetic analysis}

Combined phylogenetic analyses were performed under likelihood (ML) optimization and the GTR + G substitution model, using RAxML [24] with 500 bootstrap replicates. Bayesian analyses were conducted in MrBayes v. 3.2 [25], using the default two runs and four chains (one cold and three heated), with uniform priors on most 
parameters. Model parameters were unlinked, posterior probabilities of tree topologies were estimated from both partitions (the plastid and the mitochondrial data), and trees were sampled every 10000th generation. Burn-in and convergence were assessed using Tracer v. 1.5 [26]. Convergence was usually achieved after $4 \times 10^{6}$ generations. We used $50 \%$ majority rule consensus trees to assess posterior probabilities for nodes of interest, and the 5000 trees with the highest likelihood were used for ancestral reconstructions. All analyses were run using the Cipres Science Gateway servers [27].

\section{Ancestral character reconstruction and correlated trait analyses}

The sexual system (dioicous, state 0 , or monoicous, state 1) of each species was scored from the literature or personal observations [28-33]. Sixty-five of the $\sim 110$ known monoicous species were sampled ( 60\%), and 33 of 54 known dioicous species $(\sim 61 \%)$. Trees were rooted on Leiosporoceros, the sole genus of the Leiosporocerotaceae, which based on outgroup-rooting is sister to all other hornworts [23]. This was preferable to outgroup comparison because two of the traits that we scored lack homologues in the vascular plants: Spore diameter (small, $<35 \mu \mathrm{m}$, state 0 , or large, $>35 \mu \mathrm{m}, 1$ ) and antheridium number per chamber (low, $<9$, state 0 , or high, >10,1). Average mature spore diameters were taken from relevant literature [28-33]. The third trait, chromosome number, was taken from [34,35] and mapped onto the phylogeny.

Ancestral reconstruction relied on ML as implemented in Mesquite using the Markov 1- or 2- parameter

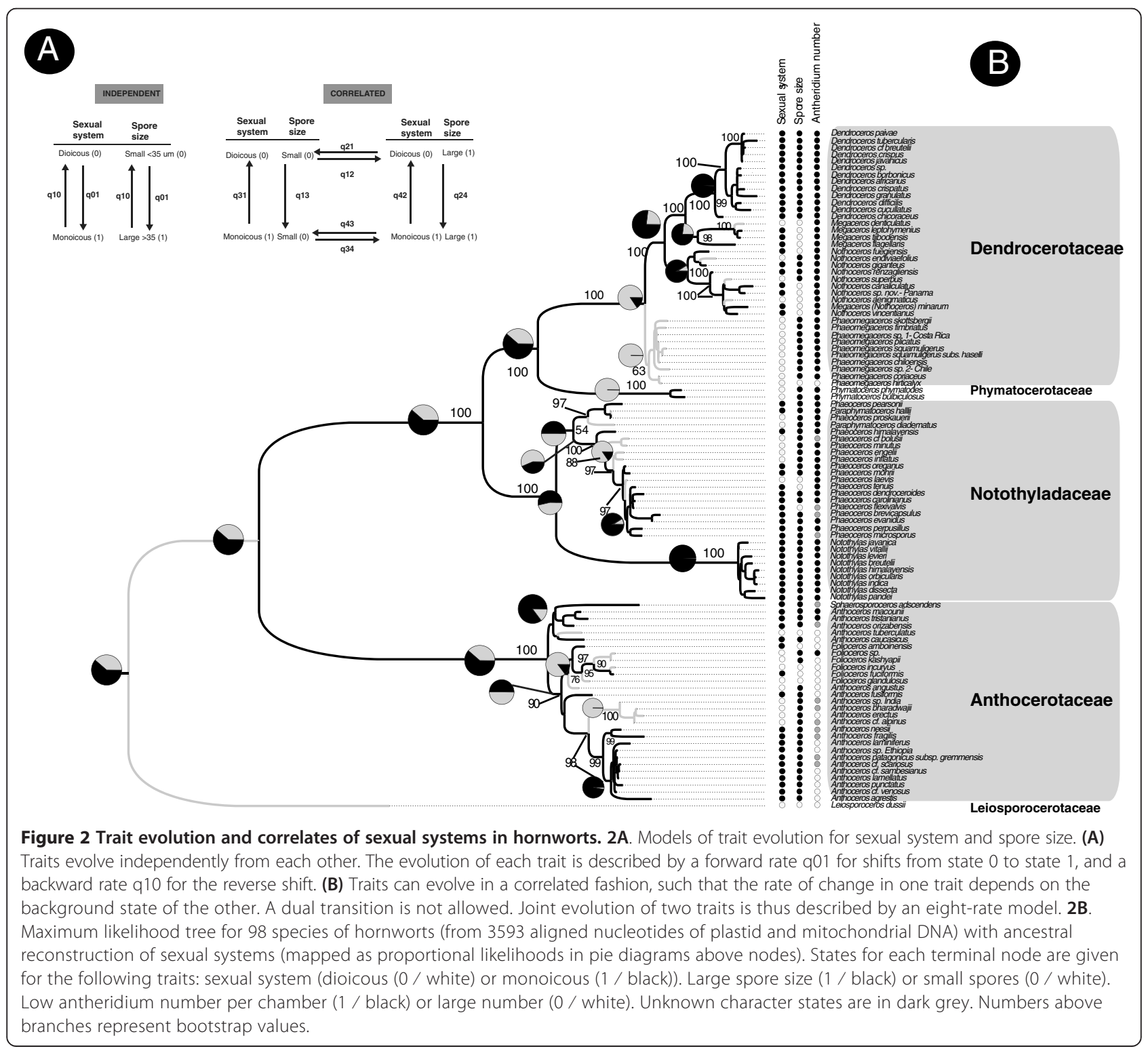


models [36] and the highest likelihood tree from RAxML. To test the null hypothesis of equal transition frequencies between the two sexual systems, we performed a likelihood ratio test (LRT) that compared the likelihood of a 1-parameter model of equal transition rates (called q) with a 2-parameter, asymmetric model, which allows separate rates of transitions to monoicy and to dioicy [37]. Test significance was evaluated based on a $\chi^{2}$ distribution with 1 degree of freedom. We also reconstructed the ancestral conditions of spore size and antheridium number using a 1-parameter model or a 2parameter model.

To test for associations between sexual system and either spore diameter or antheridium number, we used a ML approach in the DISCRETE module of BayesTraits [38]. The pair of traits was analyzed successively, using two models: a 4-rate model describing independent evolution of traits, and an 8-rate model describing correlated evolution (Figure 2A). Using the 5000 highestlikelihood trees from the Bayesian analyses, BayesTraits calculated likelihood scores for both of these models, showing how well the model fit the data. A LRT was then performed using the equation $\mathrm{LR}=-2$ ( $\mathrm{L}$ (dependent model) - L (independent model)) and a $\chi^{2}$ distribution, with four degrees of freedom (following [38]). If a trait association was barely significant we conducted 1000 simulations in Mesquite with 10 iterations for the likelihood search to obtain a $p$ value; for trait associations that were significant, we tested hypotheses about conditional evolution and the temporal order of trait acquisition by comparing the likelihood estimates of two correlated-evolution models: one in which all transition rates were allowed to vary and one that constrained the two transition rates to be equal (Figure 2A). Contingency tests involved restricting one rate to be equal to the other, re-running the analysis, and performing a LRT of the two models, using a $X^{2}$ distribution with one degree of freedom [38].

\section{Character state-associated diversification}

The diversification rate associated with each character state and the transition rates between character states have an impact on the distribution of binary traits $[39,40]$. We used the binary-state speciation and extinction (BiSSE) modelling approach to estimate transition rates among character states and character-associated diversification rates $[39,40]$. Our dataset includes 27 to $100 \%$ of the extant species in each of the hornwort genera (all of which have more than one species); sparse species sampling is a problem for all diversification modelling, and is also a problem in the present study. BiSSE model analyses were carried out in Mesquite using the diversitreeRpackage (http://www.zoology.ubc. $\mathrm{ca} /$ prog/diversitree/).

\section{Results and discussion}

The combined plastid and mitochondrial alignment had 3593 nucleotide positions of which the mat $\mathrm{K}$ gene and portions of the trnK intron comprised 700-1000 positions; we excluded the intron in the nad5 gene (Additional file 2: Matrix S1). Topologies from the individual partitions were congruent for most clades, although with less resolution in the tree obtained from the slow evolving nad5 alone. A ML tree from the combined data matrix has solid bootstrap support for most major hornwort clades except Phaeomegaceros, and its topology is consistent with previous phylogenetic reconstructions except for two novel findings: The nested position of Folioceros and Sphaerosporoceros within Anthoceros and the placement of two species of Paraphymatoceros ( $P$. hallii and the type of the genus name, $P$. diadematus) as a clade nested within Phaeoceros along with the Californian P. pearsonii and P. proskauerii (Figure 2B). Megaceros minarum is embedded in Nothoceros, and the species will need to be transferred to that genus.

Figure 1 shows the distribution of the four basic sexual systems in embryophytes, namely dioicy/monoicy in haploid-dominants and dioecy/monoecy in diploiddominant lineages (cf. Introduction). The evolution of heterospory in some ferns and the ancestor of all seed plants led to exclusively unisexual gametophytes, the ecological role of which is completely different from that of the free-living unisexual or bisexual gametophytes of the three haploid-dominant plant lineages. If earlydiverging ferns and fern-like lineages had monoicous gametophytes, then the ancestral condition in hornwort gametophytes probably also was monoicous. The ancestral condition of the hornwort sporophyte is clear: All hornworts, ferns, and lycophytes have monoecious sporophytes.

Our ancestral state reconstruction of sexual systems under a 2-rate model suggests that monoicy may have predominated in the gametophytes of early hornworts (Figure 2B), and this sexual system is inferred as ancestral in Dendroceros, Megaceros, Notothylas, Nothoceros and Anthocerotaceae (Figure 2B). Dioicy is reconstructed as the ancestral condition for Phymatoceros and Phaeomegaceros (Figure 2B). Under ML optimization, the rate of transition from dioicy to monoicy was 98.38, and the backward rate was 56.25 (Table 1). Independent reversals from monoicy to dioicy were inferred for Megaceros (1 reversal), Nothoceros (3) and Phaeoceros (1) (Figure 2B), with at least two transitions from dioicy to monoicy in Folioceros (Figure 2B).

Of the trait correlations tested, only spore size was correlated with transitions in sexual system: A contingency test revealed that transitions to dioicy were more likely in species with small spores as shown by the rate q31 of 199.72 compared with the rate q42 of 11.93 for 
Table 1 Reconstruction of trait evolution using a maximum likelihood approach

\begin{tabular}{|c|c|c|c|c|c|c|}
\hline \multirow[b]{2}{*}{ Trait } & \multicolumn{2}{|c|}{ State } & \multicolumn{4}{|c|}{ Models } \\
\hline & & & 1-rate & 2-rate & - log-likelihood- 1-rate & - log-likelihood- 2-rate \\
\hline Sexual system & Dioicous (0) & Monoicous (1) & 62.08 & $98.38,56.25$ & 53.34 & 52.28 \\
\hline Spore size & Small (0) & Large $(>35 \mu \mathrm{m})(1)$ & 49.32 & $118.42,53.92$ & 50.84 & 49.30 \\
\hline Antheridium number & 1-9 per chamber (0) & More than 10 (1) & 7.66 & $5.83,13.59$ & 19.17 & 18.64 \\
\hline
\end{tabular}

Log-likelihoods associated with the reconstruction of trait evolution for two competing explicit evolutionary models (see text for details) in hornworts. The two models (a single rate vs. a 2-rate model) were not significant different using a likelihood ratio test. The 2-rate values are given for a forward transition from state 0 to 1 , followed by the backward rate ( 1 to 0$)$.

transitions to dioicy in species with large spores (Table 2). An analysis using 5000 Bayesian trees indicated that the correlation between sexual system and spore diameter was barely significant $(\chi 2=8.31,4 \mathrm{df})$, while the 1000 simulations yielded a significant correlation (-log-likelihood -100.19 dependent model; -94.20 independent model, $p=0.01$; Table 2). Evolutionary changes in antheridium number per chamber were unrelated to spore size. Large spores are the ancestral conditions in hornworts with at least 12 transitions to small spores and no reversals (Figure 2B). Many antheridia per chamber are found in the single species of Leiosporoceros and the $\sim 80$ species of Anthoceros (22 of them included here); few (1-8) antheridia per chamber are found in the Phymatocerotaceae/Dendrocerotaceae clade and some species of Anthoceros. For both traits, the unconstrained 2-rate model had a higher likelihood than the 1-rate model (Table 1), albeit not significantly so.

Bias in the estimation of transition rates in sexual systems is introduced when the sexual systems experience different rates of diversification $[39,40]$. Our BiSSE analyses, however, showed that while the transition rate from dioicy to monoicy was 4.2 higher than vice versa $(\mathrm{q} 01=120.00 ; \mathrm{q} 10=28.17)$, the speciation rates in monoicous and dioicous clades were similar $\left(\lambda_{0}=265.34\right.$; $\left.\lambda_{1}=275.93\right)$. Monoicous groups accordingly had a higher extinction rate $\left(\mu_{0}=182.49 ; \mu_{1}=294.11\right)$. The slightly higher number of extant monoicous species (60\% vs.
$40 \%$ dioicous species) may thus be entirely due to frequent transitions to monoicy.

In species with genetic sex determination, transition to monoicy may follow genome duplication, because genome doubling may disrupt the strict segregation of the male and female sex chromosomes (Introduction). Only twenty species of hornworts have had their chromosomes counted, some repeatedly, resulting in 65 counts $[34,35]$. Sixteen of the twenty species are represented in our phylogeny (Additional file 3: Figure S1). So far, a single natural polyploid has been reported, the monoicous Anthoceros punctatus [17]. This fits the expected association of monoicy and polyploidy, but is insufficient to decide whether such an association holds across hornworts.

Our finding that lineages with small spores transition to dioicy more readily than those with large spores matches the situation in mosses, although in that clade this was only true if spore diameter was coded as a discrete character, but not when it was coded as a continuous character [12]. Crawford et al. [12] argued that evolutionary transitions to separate sexes might be easier if sporophytes produce small spores that might travel further. However, release height and wind speed probably override the importance of spore diameter in determining dispersal range [41], and it is also unclear why further dispersal might be more beneficial for dioicous species than monoicous ones. In hornworts, the

Table $\mathbf{2}$ Likelihood ratio values for analyses of trait correlation

\begin{tabular}{|c|c|c|c|c|}
\hline Trait & $\begin{array}{l}\text { Change to large spores } \\
\text { conditional upon sexual system } \\
\text { state }\end{array}$ & $\begin{array}{l}\text { Change to small spores } \\
\text { conditional upon sexual system } \\
\text { state }\end{array}$ & $\begin{array}{l}\text { Change to monoicy } \\
\text { conditional upon spore size } \\
\text { state }\end{array}$ & $\begin{array}{l}\text { Change to dioicy } \\
\text { conditional upon spore size } \\
\text { state }\end{array}$ \\
\hline $\begin{array}{l}\text { Spore } \\
\text { size }\end{array}$ & $q 12=q 34$ & $q 21=q 43$ & $q 13=q 24$ & $\mathrm{q} 31=\mathrm{q} 42$ \\
\hline \multirow[t]{2}{*}{ Rates } & $q 12=70.44$ & $q 21=19.56$ & $q 13=277.5$ & q31 = 199.72 \\
\hline & $q 34=69.35$ & $q 43=45.59$ & $q 24=35.18$ & $q 42=11.93$ \\
\hline$L(U)$ & -92.92 & -92.92 & -92.92 & -92.92 \\
\hline$L(C)$ & -93.37 & -93.58 & -94.85 & -95.81 \\
\hline$x^{2}$ & 0.89 & 1.30 & $3.85^{*}$ & $5.77^{*}$ \\
\hline
\end{tabular}

The meaning of the rate of transition between states (for example q12, q34) is shown in Figure 2A. Likelihood ratio values for analyses of correlations and tests of contingent evolution and temporal order between a life-history trait (spore size) and sexual system using Discrete. $L(U)$ represents the likelihood of the unconstrained rate estimates, $L(C)$ the likelihood when rates are set to be equal. Likelihood values were tested over a $x^{2}$ distribution with one degree of freedom. ${ }^{*} \mathrm{P}<0.05$. The only highly significant correlation was a gain of dioicy in lineages with small spores $\left(x^{2}=5.77\right)$. A gain of monoicy in lineages with small spores was barely significant $\left(x^{2}=3.85\right)$. 
sporophytes elongate by means of a basal meristem, with mature sporangium length varying from $2-3 \mathrm{~mm}$ in some species of Notothylas to $>10$ centimeters in species of Anthoceros, Megaceros, and Nothoceros. A more plausible explanation for the correlation between having small spores and transition to dioicy therefore is a resource allocation trade-off, such that larger numbers of spores can only be produced when each spore has a small diameter. Once a lineage has small spores, this may facilitate its transition to separate sexes because female thalli producing more numerous spores create a denser spore shadow, permitting sperm to more easily reach thalli of the opposite sex. Data on spore number per capsule so far are only available for Anthoceros agrestis (with large spores) [42], and experiments are required to test the above-proposed hypothesis.

In monoicous species of hornworts, the antheridia tend to develop earlier than the archegonia [14], minimizing intra-gametophyte fertilization and perhaps reducing inbreeding and the accompanying selection for dioicy. Lastly and importantly, our study of sexual system switches in hornworts, together with the data on such switches available for liverworts $[18,19]$ and mosses $[43,44]$, makes clear that suggestions of dioicy being ancestral in "bryophytes" (at the time assumed to be a monophylum) and of monoicy being the derived sexual system [2] need to be put to rest. Instead, sexual systems in the three lineages of haploid-dominant land plants are highly labile, and there is no single preferential direction. Given the under-sampling in all phylogenies used in studies of sexual system evolution to date, back and forth transitions between dioicy and monoicy probably are still underestimated. Quantitative and experimental studies of the apparent trade-off between spore diameter, spore number, and spore shadows are much needed.

\section{Conclusions}

A phylogeny for 98 of the 200 species of hornworts, representing roughly equal proportions of their monoicous and dioicous species, implies many shifts between monoicy and dioicy, terms that refer to the sexual systems of haploid tissues (compare Figure 1 for the distribution of the four main sexual systems in land plants). Different from mosses, the transition rate from dioicy to monoicy in hornworts exceeds that in the opposite direction, while diversification rates do not differ with sexual system. Trait correlation analyses revealed that transitions to dioicy are more likely in species with small spores, while the opposite is not the case. If smaller spores can be produced in larger numbers, smallerspored species may enjoy denser spore shadows, ensuring successful fertilization because of shorter distances between male and female gametophytes. However, we found no evidence of increased antheridium-per- chamber numbers in dioicous species. A correlation between monoicy and polyploidy apparently plays a small role in hornworts.

\section{Endnote}

a/http://api.ning.com/files/MUfcgT2TON39NNX3xqXMe1AuDyInYb-Bucg5pdmTT9nuFWp27Btbi/KU1bsG9aU8t5OEjIIw8QJqaMs4qIlnbQ*xZ1mKSP1x/Hornwort_matK_RBGE_PROTOCOLv1.0.pdf).

\section{Additional files}

Additional file 1: Table S1. Voucher and GenBank information. List of species used in this study including their author names, herbarium vouchers, and GenBank accession numbers for all sequences.

Additional file 2: Matrix S1 DNA alignment, morphological matrix and tree used in the study. NEXUS file of the alignment for 98 species of hornworts (from 3593 aligned nucleotides of plastid and mitochondrial DNA), a morphological matrix and maximum likelihood tree.

Additional file 3: Figure S1. Chromosome number and evolution of sexual systems in hornworts. Maximum likelihood tree for 98 species of hornworts (from 3593 aligned nucleotides of plastid and mitochondrial DNA) with ancestral reconstruction of sexual systems (mapped as proportional likelihoods in pie diagrams above nodes). States for each terminal node are given for the following traits: sexual system (dioicous (0 / white) or monoicous ( 1 / black)). Chromosome counts are mapped onto the tree for monoicous species (black stars) and dioicous species (grey stars). Anthoceros punctatus has the karyotype of a monoicous species and is a natural polyploid. Inset: The karyotypes of the dioicous Phymatoceros bulbiculosus from Portugal and of the monoicous Nothoceros vincentianus from Peru (listed as Megaceros sp. in the original paper, later identified by Proskauer as Megaceros vincentianus). Dioicous species typically have four chromosomes and one UN sex chromosomes (the U chromosome slightly larger). Monoicous species typically have 5 large chromosomes with numerous (1-5) " $m$ " or accessory chromosomes that can vary within a single gametophyte (modified from [17], all chromosomes drawn at the same scale).

\section{Competing interests}

The authors declare that they have no competing interests.

\section{Authors' contributions}

JCV performed DNA sequencing and alignment; JCV analyzed data; JCV and SSR wrote the paper; JCV and SSR designed research; both authors read and approved the final manuscript.

\section{Acknowledgements}

We thank, D. C. Cargill (Australian National Herbarium, CANB), S.

Chantanaorrapint (Songkla University, Thailand), B. Goffinet (University of Connecticut), K. Hylander (Stockholm University), T. Peng and R.-L. Zhu (Herbaria of East China Normal University), S. Pressel and J.G. Duckett (Natural History Museum, London), J. Shevock (California Academy of Sciences), and H. Stieperaere (National Botanical Garden of Belgium for plant material; D. C. Cargill for a sequences of A. fragilis; L. L. Forrest and A. Forrest (Royal Botanical Garden Edinburgh) for the matK primer and laboratory support, and N. Cusimano in our lab in Munich for providing an R script for Figure 1. Three anonymous reviewers provided constructive comments that improved the manuscript. Financial support came from the DFG grant RE-603/14-1 and SYNTHESYS GB-TAF-1893.

Received: 4 July 2013 Accepted: 16 October 2013

Published: 2 November 2013 


\section{References}

1. Bateman R, DiMichelle WA: Heterospory: the most iterative innovation in the evolutionary history of the plant kingdom. Biol Rev 1994, 69:345-417

2. Wyatt R, Anderson LE: Breeding systems in bryophytes. In The experimental biology of bryophytes. Edited by Dyer AF, Duckett JG. London: Academic; 1984:39-64.

3. Frey W, Stech M: Marchantiophyta, Bryophyta, Anthocerotophyta. In Syllabus of Plant Families - Engler's Syllabus der Pflanzenfamilien, 13ed. Part 3: Bryophytes and seedless Vascular Plants. Edited by Frey W. Berlin: Borntraeger; 2009:13-115.

4. Von Konrat M, Söderström L, Renner MAM, Hagborg A, Briscoe L, Engel JJ: Early land plants today (ELPT): how many liverwort species are there? Phytotaxa 2010, 9:22-40.

5. Magill RE: Moss diversity: new look at old numbers. Phytotaxa 2010, 9:167-174

6. Smith A, Pryer KM, Schuettpelz E, Korall P, Schneider H, Wolf PG: A classification for extant ferns. Taxon 2006, 55:705-731.

7. Renner SS, Ricklefs RE: Dioecy and its correlates in the flowering plants. Am J Bot 1995, 82:596-606.

8. Qiu Y, Li L, Wang B, Chen Z, Knoop V, et al: The deepest divergences in land plants inferred from phylogenomic evidence. Proc Natl Acad Sci USA 2006, 103:15511-15516.

9. Taylor PJ, Eppley SM, Jesson LK: Sporophytic inbreeding depression in mosses occurs in species with separate sexes but not in a species with combined sexes. Am J Bot 2007, 94:1856-1859.

10. Eppley SM, Taylor PJ, Jesson KL: Self-fertilization in mosses: a comparison of heterozygote deficiency between species with combined versus separate sexes. Heredity 2007, 98:38-44.

11. Perroud PF, Cove DJ, Quatrano RS, McDaniel SF: An experimental method to facilitate the identification of hybrid sporophytes in the moss Physcomitrella patens using fluorescence tagged lines. New Phytol 2011, 191:301-306.

12. Crawford M, Jesson LK, Garnock-Jones PJ: Correlated evolution of sexual system and life-history traits in mosses. Evolution 2009, 63:1129-1142.

13. Renzaglia KS, Villarreal JC, Duff RJ: New insights into morphology, anatomy and systematics of hornworts. In Bryophyte Biology II. Edited by Goffinet B, Shaw AJ. Cambridge: University Press; 2009:139-171.

14. Proskauer J: Studies on the morphology of Anthoceros I. Ann Bot 1948, 12:237-265

15. Cronberg N, Natcheva R, Hedlund K: Microarthropods mediate sperm transfer in mosses. Science 2006, 313:1255.

16. Rosenstiel RN, Shortlidge EE, Melnychenko AN, Pankow JF, Eppley SM: Sex-specific volatile compounds influence microarthropod-mediated fertilization of moss. Nature 2012,489:431-433.

17. Proskauer J: Studies on Anthocerotales V. Phytomorphology 1957 7:113-135.

18. Devos N, Renner MAM, Gradstein SR, Shaw AJ, Laenen B, Vanderpoorten A: Evolutionary significance and trade-offs in life history traits associated to ecological specialization and mating systems in the liverwort genus Radula. New Phytol 2011, 192:225-236.

19. Bainard JD, Forrest LL, Goffinet B, Newmaster SG: Nuclear DNA content variation and evolution in liverworts. Mol Phyl Evol 2013, 68:619-627.

20. Såstad SM: Patterns and mechanisms of polyploid speciation in bryophytes. Regnum Vegetabile 2005, 143:317-334

21. Bachtrog D, Kirkpatrick M, Mank JE, McDaniel SF, Pires JC, Rice WR, Valenzuela N: Are all sex chromosomes created equal? Trends Genet 2011, 27:350-357.

22. Duff RJ, Villarreal JC, Cargill DC, Renzaglia KS: Progress and challenges toward developing a phylogeny and classification of the hornworts. Bryologist 2007, 110:214-243.

23. Villarreal JC, Renner SS: Hornwort pyrenoids, a carbon-concentrating mechanism, evolved and were lost at least five times during the last 100 million years. Proc Natl Acad Sci USA 2012, 109:18873-18878.

24. Stamatakis $A$, Hoover $P$, Rougemont J: A rapid bootstrap algorithm for the RAxML Web-servers. Syst Biol 2008, 75:758-771. doi:10.1080/ 10635150802429642

25. Ronquist F, Teslenko M, Van der Mark P, Ayres DL, Darling A, Höhna S, Larget B, Liu L, Suchard MA, Huelsenbeck JP: MrBayes 3.2: efficient Bayesian phylogenetic inference and model choice across a large model space. Syst Biol 2012, 61:539-542.
26. Rambaut A, Drummond AJ: Tracer v. 1.5. 2007. Available from http://beast. bio.ed.ac.uk/Tracer.

27. Miller MA, Pfeiffer W, Schwartz T: Creating the CIPRES Science Gateway for inference of large phylogenetic trees. New Orleans: Proceedings of the Gateway Computing Environments Workshop (GCE); 2010:1-8. 14 Nov. 2010.

28. Asthana AK, Srivastava SC: Indian hornworts (a taxonomic study). Bryophyt Bibl 1991, 42:1-158.

29. Cargill DC, Fuhrer BA: Taxonomic studies of the Australian Anthocerotophyta II: the genus Phaeoceros. Fieldiana Bot 2008, 47:239-253.

30. Crandall-Stotler BJ, Stotler RE, Doyle WT, Forrest LL: Phaeoceros proskauerii sp. nov., a new species of the Phaeoceros hallii (Aust.) Prosk.- Phaeoceros pearsoniii (M. Howe) Prosk. complex and the systematic affinities of Paraphymatoceros Hässel. Fieldiana, Bot 2008, 47:213-238.

31. Chantanaorrapint S: Phaeoceros perpusillus (Notothyladaceae), a new species of hornwort from Thailand. Acta Bot Hungarica 2009, 51:29-33.

32. de Menéndez GG: Las especies de Phaeoceros (Anthocerotophyta) de América del Norte, Sud y Central; la ornamentación de sus esporas y taxonomía. Candollea 1989, 44:717-739.

33. Peng T, Zhu RL: A revision of the genus Anthoceros (Anthocerotaceae, Anthocerotophyta) in China. Phytotaxa 2013, 100:21-35.

34. Fritsch R: Index to bryophyte chromosome counts. Bryophyt Bibl 1991, 40:1-352.

35. Przywara L, Kuta E: Karyology of bryophytes. Polish Bot Studies 1995 9:1-83.

36. Maddison WP, Maddison DR: Mesquite: a modular system for evolutionary analysis, version 2.73. 2010. Available at http://mesquiteproject.org.

37. Pagel M: The maximum likelihood approach to reconstructing ancestral character states of discrete characters on phylogenies. Syst Biol 1999, 48:612-622

38. Pagel M: Detecting correlated evolution on phylogenies: a general method for the comparative analysis of discrete characters. Proc $R$ Soc Lond B Biol Sci 1994, 255:37-45

39. Maddison WP, Midford PE, Otto SP: Estimating a binary character's effect on speciation and extinction. Syst Biol 2007, 56:701-710.

40. FitzJohn RG, Maddison WP, Otto SP: Estimating trait-dependent speciation and extinction rates from incompletely resolved phylogenies. Syst Biol 2009, 58:595-611.

41. Lönnell N, Hylander K, Jonsson BG, Sundberg S: The fate of the missing spores - patterns of realized dispersal beyond the closest vicinity of a sporulating moss. PLoS One 2012, 7:e41987.

42. Bisang I: An estimate of mature spore production in Anthoceros agrestis in Switzerland. J Bryol 2001, 23:142-145.

43. McDaniel SF, Perroud J-P: Invited perspective: bryophytes as models for understanding the evolution of sexual systems. Bryologist 2012, 115:1-11.

44. McDaniel SF, Atwood J, Burleigh JG: Recurrent evolution of dioecy in bryophytes. Evolution 2013, 67:567-572.

doi:10.1186/1471-2148-13-239

Cite this article as: Villarreal and Renner: Correlates of monoicy and dioicy in hornworts, the apparent sister group to vascular plants. BMC Evolutionary Biology 2013 13:239.

\section{Submit your next manuscript to BioMed Central and take full advantage of:}

- Convenient online submission

- Thorough peer review

- No space constraints or color figure charges

- Immediate publication on acceptance

- Inclusion in PubMed, CAS, Scopus and Google Scholar

- Research which is freely available for redistribution 